\title{
Impact of a Hands-on Knee Exam Workshop on Medical Student Clinical Examination Scores
}

\author{
Mohammed Miniato, BS | Paul Schaefer, MD, PhD | David Weldy, MD, PhD
}

PRIMER. 2019;3:24.

Published: 10/18/2019 | DOI: 10.22454/PRiMER.2019.185529

\section{Abstract}

Introduction: The goal of this research project was to retrospectively evaluate the effect of a voluntary handson musculoskeletal knee exam workshop, presented to medical students in the family medicine rotation at the University of Toledo, on the outcomes of a required objective structured clinical examination (OSCE).

Methods: We analyzed student OSCE scores for both knee and back exams before (July 2011 to June 2012) and after (August 2013 to June 2015) the workshop was offered. The analysis was based on those who attended the voluntary knee exam workshop and those who did not. We compared scores between the two groups of students using two-tailed $t$ testing and $\chi^{2}$ testing, and assessed the correlation of attending the workshop to passing the knee OSCE.

Results: One hundred eighty-seven students attended the workshop and 279 did not. During the period when the workshop was offered, the overall mean score on the knee OSCE was $59.5 \%$ for the 187 who attended the workshop and $35.9 \%$ for the 116 who did not, which was significantly different $(P<.001)$. A $\chi^{2}$ test with a $=0.05$ showed that attending the workshop correlated with completing at least $70 \%$ of maneuvers acceptably during the knee OSCE $(P<.001)$.

Conclusions: Our study yielded positive outcomes on OSCE scores, comparable to other studies that investigated the effect of similar teaching techniques. Comparison of the scores of those who attended the knee workshop on the simpler back exam OSCE, in which no workshop was offered, demonstrated the efficacy of the workshop.

\section{Introduction}

The musculoskeletal exam is a major component of the patient physical, and one that seems to be routinely ignored by junior doctors. ${ }^{1}$ Several studies highlight the deficiencies in musculoskeletal education that can lead to the lack of student confidence in executing an examination correctly. ${ }^{2-5}$ Examination of the knee is unique in that it is almost totally dependent on the examiner's skill in manually manipulating the lower extremity, rather than resisting muscle contraction of an already properly positioned body part.

With the increased presence of technology in medical education, teaching by simulation has increased and shown promise in improving learning ability and transferring to actual execution of skills. ${ }^{67}$ Kneebone et al suggest that simulation be used alongside clinical learning for a more complete education. ${ }^{8}$ In fact, a meta-analysis by O'DunnOrto et al recommends the use of patient educators, interactive small group learning, and computer assisted learning together in teaching musculoskeletal exams. ${ }^{9}$ Small group interventions that teach skills with hands-on techniques have been shown to increase ability and retention of those skills compared to students who only participated in regular clerkship activities. ${ }^{10,11}$ Furthermore, using bedside training and direct feedback as a method 
of teaching the shoulder exam showed improved performance as well as an increase in student confidence in performing the maneuvers. ${ }^{12}$ We aimed to explore the benefit of similar hands-on training and feedback, in the form of a workshop, on medical student performance of the more skill-intensive musculoskeletal knee exam.

\section{Methods}

The Institutional Review Board at the University of Toledo approved this research project. A workshop covering how to complete a comprehensive musculoskeletal knee exam was offered during the first week of a 5-week rotation block. Workshop attendance was voluntary; therefore the sample and control groups were self-selected. Those who did not attend had online access to videos detailing the maneuvers that would be taught in the workshop. The workshop concentrated on maneuvers including visual and tactile observation, range of motion, joint line location and palpation, test for effusion, varus/valgus stress tests, Lachman's test, McMurray's test, patellar grind test, and Thessaly test. Students were presented a demonstration of each maneuver, then practiced each maneuver on at least three peers with hands-on instruction and personal feedback provided by physicians experienced in these techniques.

We analyzed student scores on a video-recorded objective structured clinical examination (OSCE) for both musculoskeletal knee and back exams on standardized patients administered during the last week of the rotation for those who did and did not attend the workshop. The OSCE comprises $7 \%$ of the final grade for the family medicine clerkship. The same physician scored all knee OSCEs, and a different single physician scored the back OSCEs. The OSCE is comprised of two 15-minute encounters with different standardized patients in which the student is required to obtain a full history and complete the relevant physical exam. Students were graded on a yes/no basis judged on their ability to completely perform each maneuver acceptably. We used sign-in sheets for the knee exam workshop to determine which students attended and we kept all student records in a Microsoft Excel file. We used Excel's data analysis tool to complete two-tailed $t$ testing and $\chi^{2}$ tests to compare scores and assess the relationship between a passing score and workshop attendance, respectively. We used scores of the back OSCE and scores before initiation of the workshop for comparison.

\section{Results}

We collected data on 187 students who attended the workshop and 116 who did not. We separated the data on 163 students evaluated when the workshop was not offered and used it as a comparison. The overall mean score on the knee OSCE was $59.5 \%$ for those 187 who attended the workshop and $35.9 \%$ for the 116 who did not, indicating that those who attended the knee exam workshop did significantly better on the knee OSCE $(P<.001)$. The overall mean scores on the back OSCE were $51.2 \%$ for those who attended the workshop, and $48.6 \%$ for those who did not, showing no significant difference in performance $(P=.216)$. Those who attended the knee workshop performed eight of the 10 graded maneuvers correctly at significantly higher rates than those who did not (Figure 1). While knee OSCE scores decreased each year, those who attended the workshop consistently did significantly better than those who did not (Table 1). A $\chi^{2}$ test with $a=0.05$ showed that attending the workshop was correlated with completing at least $70 \%$ of maneuvers acceptably during the knee OSCE $(P<.001)$.

\section{Discussion}

The results indicate that the knee exam workshop was significantly helpful in increasing student performance on the knee OSCE. A voluntary sample group makes it possible that those who attended the workshop were more inclined to perform better in assessments, although if this were the case then the workshop group's average score for the much-simpler back OSCE would have also been higher than that of the control group. Long-term retention of the physical exam maneuvers warrants further study, as students were not followed beyond their family medicine rotations in this study.

This study confirms that hands-on teaching of physical exam skills helps students better learn the technique 
required to provide a thorough exam. Thus, it may be prudent for institutions to provide hands-on training of musculoskeletal physical exam skills, especially the knee exam, which requires physical coordination, hand-eye coordination, and tactile experience. There are minimal to no financial requirements to run a workshop, as all that is required is a room to house the workshop and a faculty member to run it. There is no need to pay standardized patients, since participating students may practice on one another, as in this study, and still see the benefit. While there is no need for institutions to upend their current curriculum, the simple addition of an optional or required workshop structured like the one described in this study could be an invaluable supplement to medical students' education.

\section{Tables and Figures}

Table 1: Comparison of Mean OSCE Scores by Year and Workshop Attendance

\begin{tabular}{|c|c|c|}
\hline Academic Year & Knee OSCE Mean Score: No Workshop & Knee OSCE Mean Score: Workshop \\
\hline $2011-2012$ & 0.623 & N/A \\
\hline $2013-2014$ & 0.414 & $0.669^{*}$ \\
\hline $2014-2015$ & 0.305 & $0.532^{*}$ \\
\hline Academic Year & Back OSCE Mean Score: No Workshop & Back OSCE Mean Score: Workshop \\
\hline $2011-2012$ & 0.671 & N/A \\
\hline $2013-2014$ & 0.483 & 0.521 \\
\hline $2014-2015$ & 0.488 & 0.505 \\
\hline
\end{tabular}

Abbreviation: OSCE, objective structured clinical examination. ${ }^{*} \mathrm{P}<0.05$.

Figure 1: Comparison of Mean OSCE Scores on Performance of Knee Exam Maneuvers Based on Workshop Attendance, 2013-2015

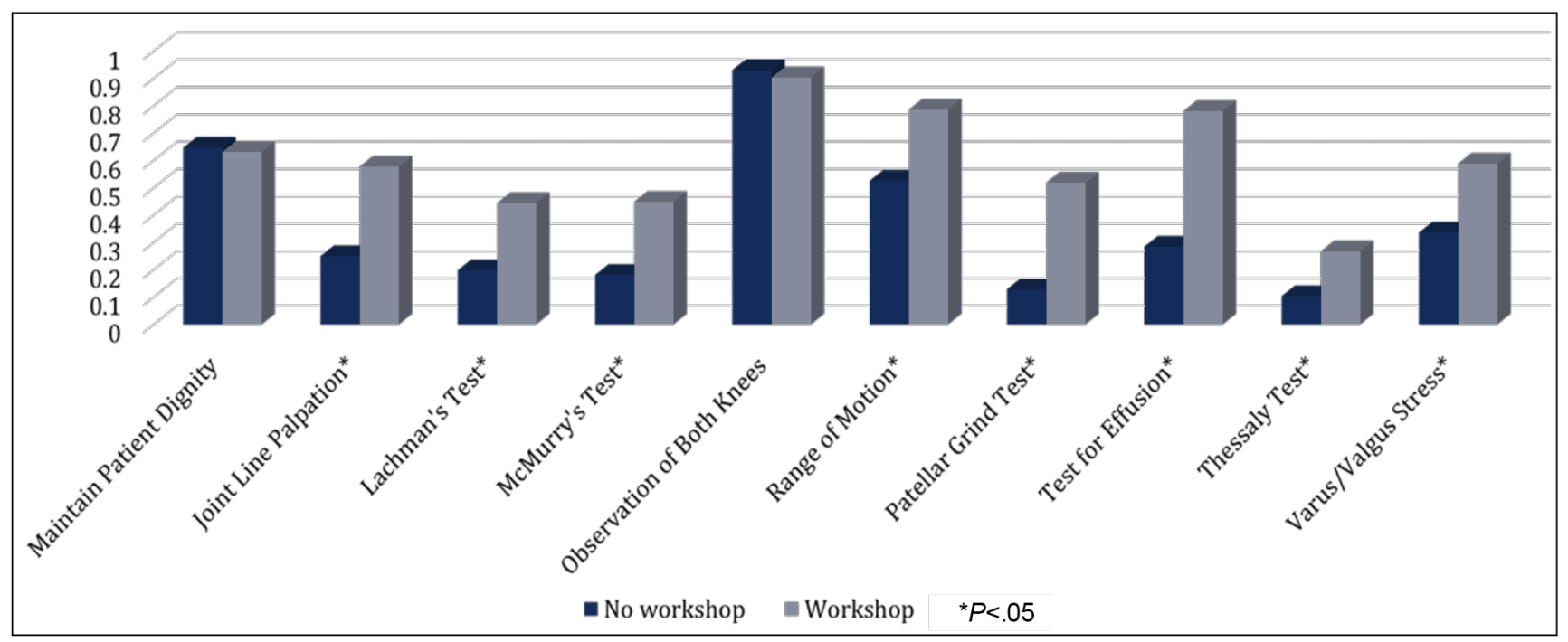

\section{Acknowledgments}

Financial Support: Financial support for this study was provided by the University of Toledo College of Medicine and Life Sciences.

Presentations: This study was presented at the following events:

- Interim Meeting of the American Medical Association, Honolulu, Hawaii. November 10, 2017

- University of Toledo College of Medicine Summer Research Forum, Toledo, OH, July 2017 


\section{Corresponding Author}

David Weldy, MD, PhD

3000 Arlington Ave. MS 1179 Toledo, OH 43614. 419-383-5573.

David.Weldy@utoledo.edu

\section{Author Affiliations}

Mohammed Miniato, BS - The University of Toledo College of Medicine and Life Sciences, Toledo, $\mathrm{OH}$

Paul Schaefer, MD, PhD - College of Medicine and Life Sciences, and Department of Family Medicine, University of Toledo Toledo, $\mathrm{OH}$

David Weldy, MD, PhD - College of Medicine and Life Sciences, and Department of Family Medicine, University of

Toledo, Toledo, $\mathrm{OH}$

\section{References}

1. Sirisena $D$, Begum H, Selvarajah M, Chakravarty K. Musculoskeletal examination-an ignored aspect. Why are we still failing the patients? Clin Rheumatol. 2011;30(3):403-407. https://doi.org/10.1007/s10067-010-1632-y

2. Day CS, Yeh AC, Franko O, Ramirez M, Krupat E. Musculoskeletal medicine: an assessment of the attitudes and knowledge of medical students at Harvard Medical School. Acad Med. 2007;82(5):452-457.

https://doi.org/10.1097/ACM.0b013e31803ea860

3. Bishop JY, Awan HM, Rowley DM, Nagel RW. Development and validation of a musculoskeletal physical examination decision-making test for medical students. J Surg Educ. 2013;70(4):451-460. https://doi.org /10.1016/j.jsurg.2013.03.011

4. Stansfield RB, Diponio L, Craig C, et al. Assessing musculoskeletal examination skills and diagnostic reasoning of 4th year medical students using a novel objective structured clinical exam. BMC Med Educ. 2016;16(1):268. https://doi.org/10.1186/s12909-016-0780-4

5. Truntzer J, Lynch A, Kruse D, Prislin M. Musculoskeletal education: an assessment of the clinical confidence of medical students. Perspect Med Educ. 2014;3(3):238-244. https://doi.org/10.1007/s40037-014-0124-1

6. Bagai A, O'Brien S, Al Lawati $\mathrm{H}$, et al. Mentored simulation training improves procedural skills in cardiac catheterization: a randomized, controlled pilot study. Circ Cardiovasc Interv. 2012;5(5):672-679. https://doi.org /10.1161/CIRCINTERVENTIONS.112.970772

7. Zhang MY, Cheng $X, X u A D$, Luo LP, Yang $X$. Clinical simulation training improves the clinical performance of Chinese medical students. Med Educ Online. 2015;20(1):28796. https://doi.org/10.3402/meo.v20.28796

8. Kneebone RL, Scott W, Darzi A, Horrocks M. Simulation and clinical practice: strengthening the relationship. Med Educ. 2004;38(10):1095-1102. https://doi.org/10.1111/j.1365-2929.2004.01959.x

9. O'Dunn-Orto A, Hartling L, Campbell S, Oswald AE. Teaching musculoskeletal clinical skills to medical trainees and physicians: a Best Evidence in Medical Education systematic review of strategies and their effectiveness: BEME Guide No. 18. Med Teach. 2012;34(2):93-102. https://doi.org/10.3109/0142159X.2011.613961

10. Perrig M, Berendonk $C$, Rogausch A, Beyeler $C$. Sustained impact of a short small group course with systematic feedback in addition to regular clinical clerkship activities on musculoskeletal examination skills-a controlled study. BMC Med Educ. 2016;16(1):35. https://doi.org/10.1186/s12909-016-0554-z

11. Lawry GV II, Schuldt SS, Kreiter CD, Densen P, Albanese MA. Teaching a screening musculoskeletal examination: a randomized, controlled trial of different instructional methods. Acad Med. 1999;74(2):199-201. https://doi.org/10.1097/00001888-199902000-00020

12. Blohm $\mathrm{M}$, Krautter $\mathrm{M}$, Lauter $\mathrm{J}$, et al. Voluntary undergraduate technical skills training course to prepare students for clerkship assignment: tutees' and tutors' perspectives. BMC Med Educ. 2014;14(1):71. https://doi.org/10.1186/1472-6920-14-71

Copyright $(2019$ by the Society of Teachers of Family Medicine 